\title{
Entwurf von zeitkontinuierlichen $\Sigma \Delta$-Modulatoren - Äquivalenz zwischen zeitkontinuierlichen und zeitdiskreten Systemen
}

\author{
J. Anders ${ }^{1}$, W. Mathis ${ }^{2}$, and M. Ortmanns ${ }^{2}$ \\ ${ }^{1}$ Institut für Theoretische Elektrotechnik, Leibniz Universität Hannover, Appelstraße 9A, 30167 Hannover, Deutschland \\ ${ }^{2}$ Institut für Mikrosystem Technologie, Albert Ludwigs Universität, Georges-Koehler-Allee 102, 79110 Freiburg, Deutschland
}

\begin{abstract}
Zusammenfassung. Im vorliegenden Artikel wird eine vollstängige, d.h. unter Einbeziehung des Eingangssignals, Äquivalenz zwischen zeitkontinuierlichen und zeitdiskreten $\Sigma \Delta$-Modulatoren im Zustandsraum hergeleitet. Es wird ebenfalls gezeigt, wie eine wichtige Nichtidealität, das sog. "excess loop delay", in den Berechnungen berücksichtigt werden kann. Die dargestellte Methode dient dazu, den Entwurf und dabei vor allem die Stabilitätsuntersuchungen, von zeitkontinuierlichen $\Sigma \Delta \mathrm{s}$ auf die bereits empirisch intensiv erforschten zeitdiskreten Systeme zurückzuführen.
\end{abstract}

\begin{abstract}
In the article at hand a complete equivalency, i.e. including the input signal, between continuous-time and discrete-time $\Sigma \Delta$-modulators in state-space is derived. In addition, it is shown, how one can incorporate the important non-ideality of "excess loop delay" into the formalism. The method introduced is supposed to facilitate the design, especially the stability analysis part of the design, of continuoustime $\Sigma \Delta$ s by making use of the empirically thoroughly examined discrete-time systems.
\end{abstract}

\section{Einleitung}

Es ist eine allgemein bekannte Tatsache, dass, vornehmlich aus Kostengründen, in den letzten Jahrzehnten ein Trend zur Verschiebung von der Analogdomäne hin zu digitalen Realisierungen in modernen VLSI-Prozessen erkennbar ist. Aufgrund der damit verbundenen ständig wachsenden Anforderungen an die Leistungsfähigkeit von Analog-/Digital Umsetzern in Bezug auf Bandbreite und "dynamic range", sowie des Wunsches analoge und digitale Schaltungsteile in dem selben, kostenünstigen CMOS-Prozess realisieren zu können, ist in den letzten zwei Jahrzehten das Interesse an $\Sigma \Delta$-Umsetzern rapide gestiegen. Diese Um-

Correspondence to: J. Anders

(anders@tet.uni-hannover.de) setzer erreichen aufgrund ihrer Toleranz gegenüber Nichtidealitäten wie Bauelementerauschen und Komponentenmismatch auch in für analoge Zwecke eher ungeeigneten CMOS-Implementierungen mit niedrigen Versorgunsspannungen und schlecht definierten Transistorparametern erstaunliche Ergebnisse, indem sie einen Großteil der Funktionalität in die digitalen Blöcke verlagern (digitale Dezimation) und somit effizient die Konzepte "Rückkopplung" und "Überabtastung" verbinden. Während bis vor wenigen Jahren $\Sigma \Delta$-Modulatoren meist zeitdiskret in Form von switched-capacitor Schaltungen realisiert wurden, liessen die enorm wachsenden Bandbreiteanforderungen das Interesse an zeitkontinuierlichen Modulatoren in den letzten zehn Jahren stark anwachsen, vgl. Ortmanns und Gerfers (2006). Dabei bedienten sich die ersten Designer von zeitkontinuierlichen Modulatoren meist des in der zwischenzeit angesammelten immensen Vorrats an Wissen über zeitdiskrete Modulatoren und der Entwurf bestand in der Regel in einer Abbildung der optimalen Parameter für den zeitdiskreten Modulator auf das zeitkontinuierliche Gegenstïck, davon ausgehend, die Optimalität bliebe durch die Transformation erhalten. In der Annahme, dass die Stabilität ausschliesslich durch ein Nicht-Überladen des Quantisierereingangs gewährleistet werden könne, wurde zur Transformation aber lediglich das Ein- Ausgangsverhalten zwischen Quantisierungsfehlereingang und Quantisierereingang, also die sognenannte Rauschübertragungsfunktion, verwendet, wobei die internen Zustandsvariablen und somit die gesamte interne Dynamik des $\Sigma \Delta$-Modulators, in der Regel komplett vernachlässigt wurden, vgl. Cherry und Snelgrove (2000), so dass hier eine genauere Untersuchung des dynamischen Verhaltens und dessen Auswirkung auf die Stabilität nötig ist. In der vorliegende Arbeit wird daher eine Äquivalenzbeziehung im Zustandsraum zwischen zeitkontinuierlichen und zeitdiskreten Modulatoren hergeleitet, die auch das Eingangssignal des Systems berücksichtigt und somit auch für Stabilitätsbetrachtungen die gewünschte Austauschbarkeit von zeitdis- 


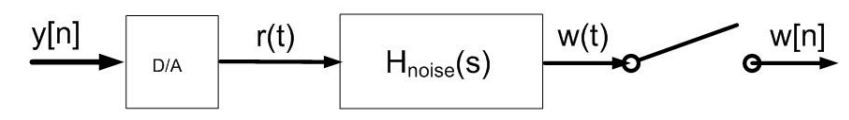

Abb. 1. Offene Kette des Rauschformungssytems.

kretem und zeitkontinuierlichem Modulator liefert.

Nach einem kurzen Rückblick auf die bisher meist verwandte Methode nach Cherry, vgl. Cherry und Snelgrove (2000), in Abs. 2 werden in Abs. 3 dann die notwendigen Äquivalenzbeziehungen im Zustandsraum hergeleitet und es wird gezeigt, wie "excess loop delay" in dem Formalismus berücksichtigt werden kann. Abschließend folgt eine Zusammenfassung und ein kurzer Ausblick.

\section{2 Äquivalenz zwischen zeitkontinuierlichen und zeitdis- kreten Modulatoren}

Wie bereits in der Einleitung erwähnt, ist ein funktionelle Äquivalenz zwischen zeitkontinuierlichen (CT-) und zeitdiskreten (DT-) $\Sigma \Delta$-Modulatroen aus einer Reihe von Gründen erstrebenswert. Ein sehr wichtiger Grund ist dabei, dass bereits auf eine relative große Erfahrung für den Entwurf zeitdiskreter Modulatoren zurückgegriffen werden kann und es daher wünschenswert ist, dieses Wissen auch für den Entwurf zeitkontinuierlicher $\Sigma \Delta \mathrm{s}$ nutzbar zu machen. Desweiteren ist auch die Simulationszeit, d.h. die Zeit die die transiente Simulaion eines Diskretzeitmodulators, bei gegebenem Simulationsintervall $\Delta T_{\text {sim }}$, benötigt wesentlich kürzer als die Simulationszeit für einen zeitkontinuierlichen Modulator. Dies kann dadurch erklärt werden, dass für die transiente Simulation eines zeitdiskreten Modulators lediglich Matrizenmultiplikationen und Additionen vonnöten sind, während für die Simulation eines zeitkontinuierlichen Modulators zusätzlich noch vektorwertige Faltungsintegrale gelöst werden müssen. Folglich gab es auch immer schon Bestrebungen, funktionell äquivalente zeitdiskrete Modulatoren für einen gegebenen zeitkontinuierlichen Modulator zu finden. Dabei beschränkten sich die meisten Autoren in der Vergangenheit auf eine Äquivalenz des Rauschformungsverhaltens zwischen den beiden Modulatoren und vernachlässigten somit das gerade für Stabilitätsbetrachtungen immens wichtige Eingangssignal. Um nun ein identisches Rauschformungsverhalten bei genulltem Eingangssignal und innerhalb der Gültigkeitsgrenzen des additiven weißen Rauschmodells für den Quantisierer zu erlangen, haben Cherry und Snelgrove (2000), gezeigt, dass eine Äquivalenz der in Abb. 1 dargestellten offenen Kette des Rauschformungssystems an den Abtastzeitpunkten $n T$ hinreichend für ein identisches Verhalten der beiden Systeme, zeitdiskreter und zeitkontinuierlicher Modulator, ist. Nach Snelgrove und Cherry sind also zwei Modulatoren genau dann äquivalent, wenn sie bei gleicher Eingangssequenz, $y[n]$ der offenen Rauschformungskette gemäß Abb. 1 das gleiche Ausgangssignal

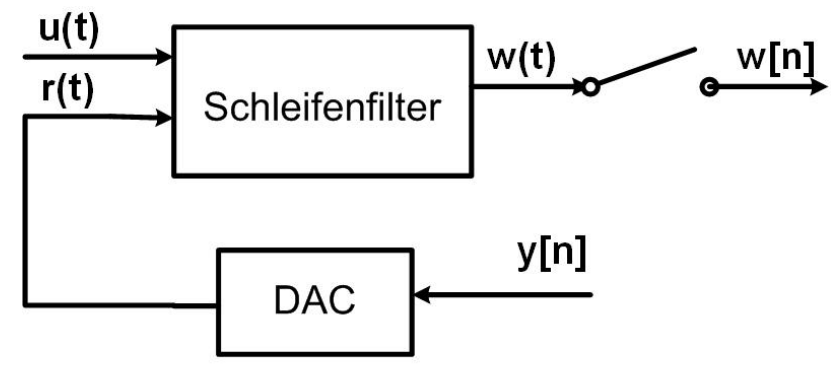

Abb. 2. Offene Kette - Zustandsraumdarstellung mit Eingangssignal.

$w[n]=w(n T)$ aufweisen. Im Zeitbereich lässt sich diese Bedingung wie folgt darstellen

$\mathcal{Z}^{-1}\left\{H_{e q}(z)\right\}=\left.\mathcal{L}^{-1}\left\{H_{D A}(s) H_{\text {noise }}(s)\right\}\right|_{t=n T_{s}}$,

wobei $H_{D A}(s)$ die Laplace-Transformierte der DACImpulsantwort und $H_{e q}(z)$ die z-Transformierte des äquivalenten zeitdiskreten Schleifenfilters ist. Ein Vorteil dieses Verfahrens ist, dass auch Nichtidealitäten wie "excess loop delay" leicht in den Formalismus eingebettet werden können. Allerdings steht diesem Vorteil der schwerwiegende Nachteil gegenüber, dass das Eingangssignal komplett vernachlässigt wird und somit der berechnete zeitdiskrete Modulator für Stabilitätsbetrachtungen ${ }^{1}$ nicht mehr äquivalent zum ursprünglichen zeitkontinuierlichen Modulator ist. Als alternatives Verfahren wird im folgenden eine Betrachtung im Zustandsraum vorgestellt, die auf Schreier und Zhang (1996) und Oliae (2003), zurückgeht, diese wird aber dahingehend erweitert, dass sowohl das Eingangssignal berücksichtigt wird, als auch eine Berücksichtigung von "excess loop delay" in dem Verfahren möglich ist.

\section{3 Äquivalenz im Zustandsraum}

Das Schleifenfilter eines zeitkontinuierlichen $\Sigma \Delta$ Modulators lässt sich im Zustandsraum wie folgt darstellen

$$
\left\{\begin{array}{l}
\dot{x}_{c}(t)=A_{c} x_{c}(t)+\left[B_{c 1} B_{c 2}\right]\left[\begin{array}{l}
u(t) \\
r(t)
\end{array}\right] \\
w(t)=C_{c} x_{c}(t)+\left[D_{c 1} D_{c 2}\right]\left[\begin{array}{l}
u(t) \\
r(t)
\end{array}\right]
\end{array} .\right.
$$

Da sich der Ausgang des DAC als Faltung einer Serie von Dirac-Impulsen, die mit der Folge $y[n]$ gewichtet ist, mit der DAC-Impulsantwort $h_{d a}(t)$ gemäß

$$
r(t)=h_{d a}(t) \star \sum_{l=0}^{\infty} y[l] \delta(t-l T)=\sum_{l=0}^{\infty} y[l] h_{d a}(t-l T)
$$

\footnotetext{
${ }^{1}$ In $\Sigma \Delta$ Modulatoren ist die Stabilitä abhängig vom Eingangssignal.
} 
schreiben lässt und die Lösung von Gl. (2) durch

$x_{c}(t)=e^{A_{c}\left(t-t_{0}\right)} x_{c}\left(t_{0}\right)+\int_{t_{0}}^{t} e^{A_{c}(t-\lambda)}\left[B_{c 1} B_{c 2}\right]\left(\begin{array}{c}u(\lambda) \\ r(\lambda)\end{array}\right) d \lambda$

gegeben ist, erhält man durch Einsetzen von Gl. (3) in Gl. (4) das zeitliche Verhalten der offenen Kette gemäß Abb. 2 als

$$
\begin{aligned}
x_{c}(t)= & e^{A_{c}\left(t-t_{0}\right)} x_{c}\left(t_{0}\right)+\int_{t_{0}}^{t} e^{A_{c}(t-\lambda)} B_{c 1} u(\lambda) d \lambda \\
& +\sum_{l=0}^{\infty} y[l] \int_{t_{0}}^{t} e^{A_{c}(t-\lambda)} B_{c 2} h_{d a}\left(\lambda-l T_{s}\right) d \lambda .
\end{aligned}
$$

Wählt man nun den Anfangszustand $x_{c}\left(t_{0}\right)$ zum Zeitpunkt $t_{0}=n T$ gemäß $x_{c}\left(t_{0}\right) \equiv x[n]$ und wertet Gl. (5) am nächsten Abtastzeitpunkt aus, d.h. $x_{c}((n+1) T) \equiv x[n+1]$, so erhält man nach der Substitution $\mu=\lambda+n T$

$$
\begin{aligned}
x[n+1]= & e^{A_{c} T} x[n]+\int_{0}^{T} e^{A_{c}(T-\mu)} B_{c 1} u(\mu+n T) d \mu \\
& +\sum_{l=0}^{\infty} y[l] \int_{0}^{T} e^{A_{c}(T-\mu)} B_{c 2} h_{d a}(\mu+(n-l) T) d \mu .
\end{aligned}
$$

Um nun den äquivalenten zeitdiskreten Modulator zu erhalten, vergleicht man das Ergebnis aus Gl. (6) mit der offenen Kette eines generischen zeitdiskreten Modulators, die in Gl. (7) dargestellt ist.

$$
\left\{\begin{array}{l}
x[n+1]=A x[n]+\left[B_{1} B_{2}\right]\left[\begin{array}{l}
u[n] \\
y[n]
\end{array}\right] . \\
w[n]=C x[n]+\left[D_{1} D_{2}\right]\left[\begin{array}{l}
u[n] \\
y[n]
\end{array}\right]
\end{array}\right.
$$

Im folgenden wird noch, um algebraische Schleifen auszuschließen, angenommen, dass der Durchgangsanteil $D_{2}$ verschwindet.

Zwei Schwierigkeiten behindern den direkten Koeffizientenvergleich zwischen Gl. (6) und Gl. (7). Zum einen taucht das Eingangssignal $u_{c}(t)$ in Gl. (6) nicht in der Form $B_{1} \cdot u_{c}[n T]$ auf und zum anderen hängt der folgende $\mathrm{Zu}$ stand im Falle des zeitkontinuierlichen Modulators, je nach Länge der Impulsantwort des DAC auch von vergangenen Ausgangswerten $y[n-k], k=0,1, \ldots, M$ ab.

Das erste Problem lässt sich durch die Einführung eines äquivalenten zeitdiskreten Eingangssignals $u_{e q}[n]$ gemäß

$u_{e q}[n]=\int_{0}^{T} e^{A_{c}(T-\mu)} B_{c 1} u(\mu+n T) d \mu$.

lösen. Wie die folgenden Überlegungen zeigen werden muss man zur Lösung des zweiten Problems eine Erweiterung des
Zustandsvektors des äquivalenten zeitdiskreten Modulators vornehmen.

Im folgenden wird angenommen, dass die DACImplulsnatwort nur im Intervall $\left[0, T_{e}\right]$, das $M+1$ Abtastintervalle umfasse, von null verschieden sei. Dann kann die unendliche Summe in Gleichung 6 wie folgt vereinfacht werden.

$$
\begin{aligned}
x[n+1]= & e^{A_{c} T} x[n]+\int_{0}^{T} e^{A_{c}(T-\mu)} B_{c 1} u(\mu+n T) d \mu \\
& +\sum_{l=n-M}^{n} y[l] \int_{0}^{T} e^{A_{c}(T-\mu)} B_{c 2} h_{d a}(\mu+(n-l) T) d \mu .
\end{aligned}
$$

Das weitere Vorgehen wird nun am Beispiel des in der Praxis wohl relevantesten Falles von $M=1$, also einer DACImpulsantwort, die in die sich in die nächste Abtastperiode hineinerstreckt, verdeutlicht und dann auf den allgemeinen Fall eines beliebigen $M$ erweitert.

Für $M=1$ erhält man

$$
\begin{aligned}
& x[n+1]= e^{A_{c} T} x[n]+u_{e q}[n]+ \\
& y[n] \int_{0}^{T} e^{A_{c}(T-\mu)} B_{c 2} h_{d a}(\mu) d \mu+ \\
& y[n-1] \int_{0}^{T} e^{A_{c}(T-\mu)} B_{c 2} h_{d a}(\mu+T) d \mu .
\end{aligned}
$$

Durch Einführung der neuen Zustandsvariablen $\xi_{1}[n] \equiv y[n-1]$ lässt sich diese Gleichung in Matrizenform darstellen und man erhält die erweiterte Zustandsraumdarstellung des zeitkontinuierlichen Modulators an den Abtastzeitpunkten gemäß

$$
\begin{aligned}
{\left[\begin{array}{c}
x[n+1] \\
\xi_{1}[n+1]
\end{array}\right]=} & {\left[\begin{array}{cc}
e^{A_{c} T} & \boldsymbol{B}_{2 n} \\
0 & 0
\end{array}\right]\left[\begin{array}{l}
x[n] \\
\xi_{1}[n]
\end{array}\right]+} \\
& {\left[\begin{array}{c}
\boldsymbol{B}_{2(n-1)} \\
1
\end{array}\right] y[n]+u_{e q}[n], }
\end{aligned}
$$

wobei die Vektoren $\boldsymbol{B}_{2 n}$ and $\boldsymbol{B}_{2(n-1)}$ folgendermaßen berechnet werden müssen

$\boldsymbol{B}_{2 n}=\int_{0}^{T} e^{A_{c}(T-\mu)} B_{c 2} h_{d a}(\mu+T) d \mu$

$\boldsymbol{B}_{2(n-1)}=\int_{0}^{T} e^{A_{c}(T-\mu)} B_{c 2} h_{d a}(\mu) d \mu$.

Äquivalenz zwischen einem zeitdiskretem und zeitkontinuirlichem Modulator besteht also offenbar genau dann, wenn 
die Zustände und Systemmatrizen des zeitdiskreten Modulators wie folgt gewählt werden

$$
\begin{aligned}
& x_{D T}[n]=\left[\begin{array}{l}
x_{c}(n T) \\
\xi_{1}(n T)
\end{array}\right] \\
& A_{D T}=\left[\begin{array}{cc}
e^{A_{c} T} & B_{2 n} \\
0 & 0
\end{array}\right] \\
& B_{2 D T}=\left[\begin{array}{c}
B_{2(n-1)} \\
1
\end{array}\right] \\
& B_{1 D T} u[n]=u_{e q}[n] \\
& C_{D T}=\left[\begin{array}{ll}
C_{c} & 0
\end{array}\right] \\
& D_{1 D T}=D_{c 1} \text {. }
\end{aligned}
$$

Im allgemeinen Fall für $M$ beliebig schreibt man zunächst Gl. (6) mit den neuen Grössen $A$ und $B_{2 l}$

$$
\begin{aligned}
A & \equiv e^{A_{c} T} \\
B_{2 l} & \equiv \int_{0}^{T} e^{A_{c}(T-\mu)} B_{c 2} h_{d a}(\mu+(n-l) T) d \mu
\end{aligned}
$$

folgendermassen um

$x[n+1]=A x[n]+u_{e q}[n]+\sum_{l=0}^{\infty} y[l] B_{2 l}$.

Anschliessend führt man die zusätzlichen Zustandsvariablen

$$
\begin{aligned}
\xi_{1}[n] & =y[n-1] \\
\xi_{2}[n] & =y[n-2] \\
\vdots & \\
\xi_{M}[n] & =y[n-M]
\end{aligned}
$$

ein und erhält so die erweiterte Zustandsraumdarstellung für den zeitkontinuierlichen Modulator an den Abtastzeitpunkten

$$
\begin{gathered}
{\left[\begin{array}{c}
x[n+1] \\
\xi_{1}[n+1] \\
\xi_{2}[n+1] \\
\vdots \\
\xi_{k}[n+1]
\end{array}\right]=\tilde{A}\left[\begin{array}{c}
x[n] \\
\xi_{1}[n] \\
\xi_{2}[n] \\
\vdots \\
\xi_{k}[n]
\end{array}\right]+\left[\begin{array}{c}
B_{2 n} \\
1 \\
0 \\
\vdots \\
0
\end{array}\right] y[n]+u_{e q}[n]} \\
w[n]=\left[\begin{array}{llll}
C_{c} & 0 & 0
\end{array}\right]
\end{gathered}
$$

mit den neu definierten Grössen

$$
\tilde{A} \equiv\left[\begin{array}{cccccc}
e^{A_{c} T} & B_{2(n-1)} & B_{2(n-2)} & \cdots & B_{2(n-(k-1))} & B_{2(n-k)} \\
0 & 0 & 0 & \cdots & 0 & 0 \\
0 & 1 & 0 & \cdots & 0 & 0 \\
0 & 0 & 1 & \cdots & 0 & 0 \\
\vdots & \vdots & \ddots & \ddots & \vdots & \vdots \\
0 & 0 & 0 & \cdots & 1 & 0
\end{array}\right]
$$

\section{Diskussion}

Es wurde eine vollständige Äquivalenz zwischen zeitkontinuierlichen und zeitdiskreten $\Sigma \Delta$-Modulatoren im Zustandsraum hergeleitet. Die vorgestellte Methode erweitert die Betrachtungen von Schreier und Zhang (1996), und Oliae (2003), da sie auch das Eingangssignal sowie "excess loop delay" berücksichtigt. Daher ist es möglich, mit Hilfe der dargestellten Methode auch Stabilitätsbetrachtungen für den zeitkontinuierlichen Modulator an seinem zeitdiskreten $\mathrm{Ge}-$ genstück durchzuführen, was in der Regel günstiger ist, da man dabei mangels eines allgemeingültigen Stabilitätskriteriums, auf transiente Simulationen zurückgreifen muss, die für einen zeitdiskreten Modulator wesentlich schneller durchgeführt werden können. Dennoch ist es für die Zukunft mehr als wünschenswert, ein solches allgemeingültiges Stabilitätskriterium zu finden. Die oben vorgestellte Methode kann dann dazu dienen, dieses Kriterium, je nachdem ob es für zeitdiskrete oder zeitkontinuierliche Modulatoren gefunden wurde, auf den jeweils anderen Modulatortyp zu übertragen.

\section{Literatur}

Ortmanns, M. und Gerfers, F.: Continuous-Time Sigma-Delta A/D Conversion, Springer, 2006.

Cherry, J. A. und Snelgrove, W. M.: Continuous-Time Delta-Sigma Modulators for High-Speed A/D Conversion, Kluwer Academic Publishers, 2000.

Oliaei, O.: Design of Continuous-Time Sigma-Delta Modulators With Arbitrary Feedback Waveform, IEEE Transactions on Circuits and Systems - II, Analog and Digital Signal Processing, vol. 50, no. 8, August, 2003.

Schreier, R. and Zhang, B.: Delta-sigma modulators employing continuous-time circuitry, IEEE Trans. Circuits Syst. I, 43, 324 332, April, 1996.

Ogata, K.: Discrete-Time Control Systems, 2nd ed., Englewood Cliffs, NJ: Prentice Hall, 1997. 